\title{
Enzyme-Powered Nanomotors with Controlled Size for Biomedical Applications
}

\author{
Jiawei Sun, Motilal Mathesh, Wei Li, and Daniela A. Wilson*(i) \\ Institute for Molecules and Materials, Radboud University, Heyendaalseweg 135, 6525 AJ Nijmegen, The Netherlands
}

\author{
Supporting Information
}

\begin{abstract}
Self-propelled motors have been developed with promising potential for medical applications. However, most of them have a size range at the microscale, which limits their further research for in vivo experiments. Previously, our group developed nanoscaled motors with a size of around $400 \mathrm{~nm}$ with several merits, for example, delivering both hydrophobic and hydrophilic drugs/proteins, using biocompatible fuels while being able to control their motion, and showing adaptive changes of their speed and navigation to changes in the environment. It is also wellknown that nanoparticles that are around $20-200 \mathrm{~nm}$ in size have advantages in overcoming cellular barriers and being internalized into cells. Therefore, lowering the size range of this stomatocyte nanomotor is crucial. However, the strict control of the size of

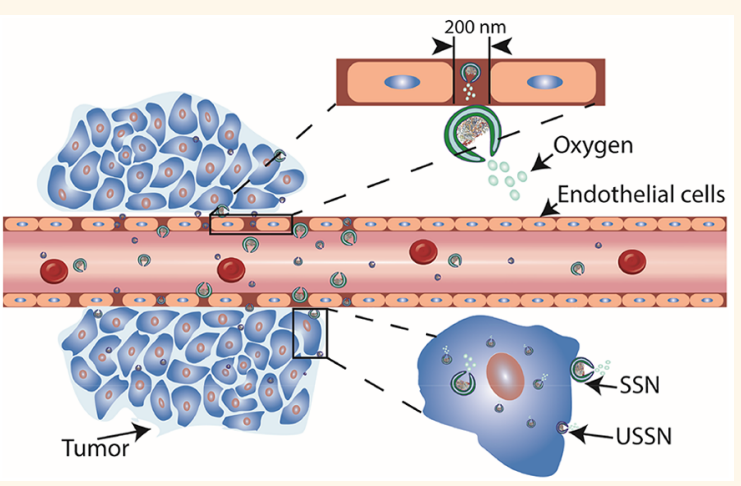
vesicles in such a low regime as well as their shape transformation into folded stomatocyte structures is not trivial. In this study, we fabricated ultrasmall stomatocyte polymersomes with the size of around $150 \mathrm{~nm}$, which could be a promising carrier for biomedical purposes. We demonstrated that the addition of PEG additive allows for both shape transformation of small polymersomes into stomatocytes and encapsulation of biologics. Biocatalyst catalase was encapsulated in the inner compartment of the nanomotor, protecting the enzyme while providing enough thrust to propel the motors. The ultrasmall stomatocyte motor system allowed propelled motion by converting $\mathrm{H}_{2} \mathrm{O}_{2}$ into $\mathrm{O}_{2}$ in the presence of only $2 \mathrm{mM}$ $\mathrm{H}_{2} \mathrm{O}_{2}$, and the velocity of motors correlated to the $\mathrm{O}_{2}$ production. Compared to small stomatocyte nanomotors, ultrasmall stomatocyte motors demonstrate enhanced penetration across the vasculature model and increased uptake by HeLa cells in the presence of fuel.
\end{abstract}

KEYWORDS: polymersomes, nanomotors, biocatalyst, cell uptake, oxygen production, biomedical applications

I nspired by natural molecular motors, ${ }^{1-3}$ scientists have been focusing on developing motors from the macro- and micro- to the nanometer scale for numerous potential applications such as nanoscale fabrication, environmental remediation, and biomedicine. ${ }^{4-10}$ Recent advances in micro/nanomotor research have made them promising tools for addressing many biomedical challenges owing to their unique features, including fast motion, high cargo loading and towing force, and ease of surface functionalization. ${ }^{11-13}$ For example, several motors have already demonstrated great potential for targeted drug delivery and cargo release efficiency, including self-propelling polymer multilayer nanorockets and TAT peptide-modified stomatocyte nanomotors. ${ }^{14,15}$ However, there are still many bottlenecks that need to be addressed, such as use of metal surfaces that are toxic to humans and the current size spectra that are not suitable for clinical trials. The size of nanoparticles is one of the most important factors determining whether the particles can pass certain biological barriers or not (e.g., blood, extracellular matrix, and cellular barriers). ${ }^{16-19}$ For instance, particles above the size range of 4-5 nm could avoid renal and hepatic clearance and have a longer blood circulation time. Meanwhile, if their sizes are smaller than $200 \mathrm{~nm}$, nanoparticles are more prone to extravasate from tumor vasculature as vascular abnormalities in tumor tissues lead to permeability of the vascular system, $^{20,21}$ allowing tumors to accumulate small particles based on the physiological principle. ${ }^{22}$ In this context, researchers have shown that smaller nanomotors present an advantage in overcoming cellular barriers and improving cellular uptake. ${ }^{23}$ For instance, researchers have developed Janus mesoporous silica nanomotors (MSNs) of less than 100 $\mathrm{nm}$ as carriers for drug delivery with controlled release. ${ }^{24}$ However, clearance of these nanomotors by the mononuclear phagocytic system (MPS) at the blood barrier is possible, due to the lack of biocompatible elements such as poly(ethylene glycol) (PEG). ${ }^{25}$ PEGylation onto the surface of nanoparticles prevents interaction between nanoparticles and with biological fluid components, such as plasma proteins, leading to reduced

Received: May 1, 2019

Accepted: August 22, 2019

Published: August 27, 2019 


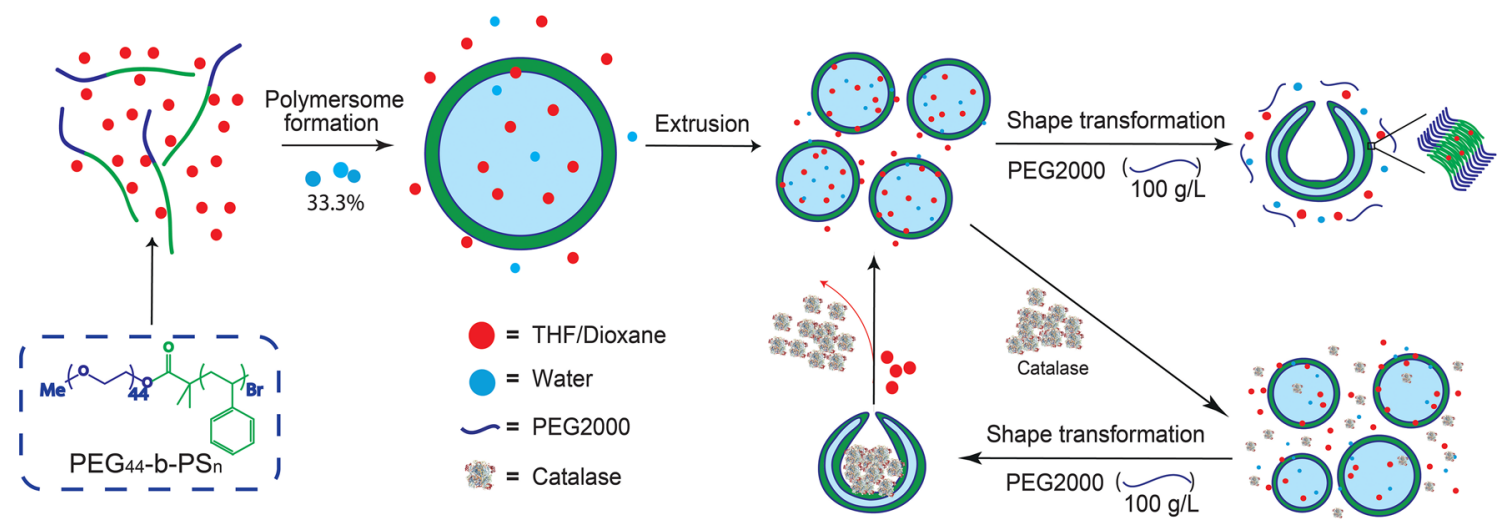

Figure 1. Scheme for the preparation of size-controlled poly(ethylene glycol)-b-polystyrene (PEG-PS) stomatocyte-like particles and enzyme-loaded nanomotors.
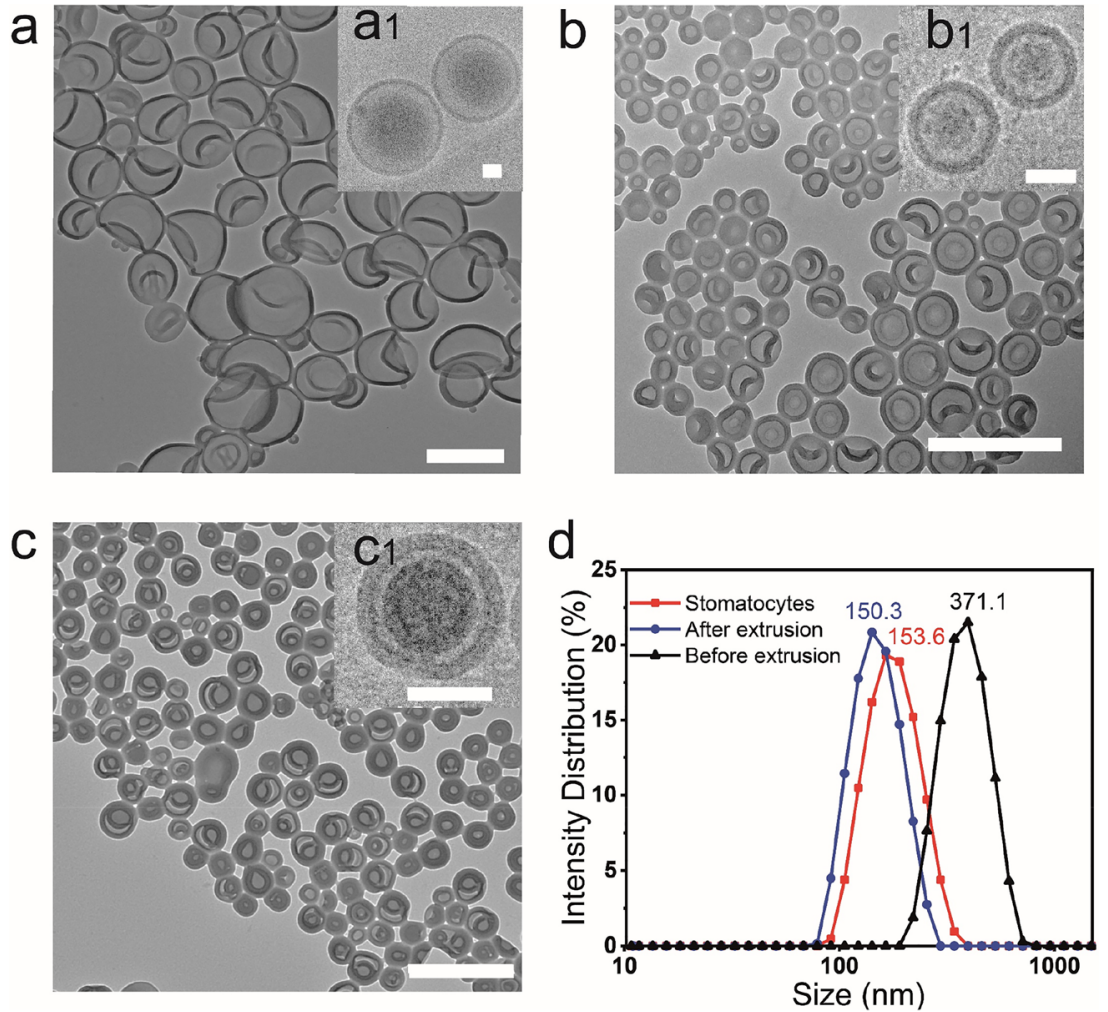

Figure 2. TEM (a,b) and cryo-TEM images $\left(a_{1}, b_{1}\right)$ of polymersomes before and after extrusion. TEM (c) and cryo-TEM images $\left(c_{1}\right)$ of sizecontrolled stomatocytes. (d) Size distribution of polymersomes before extrusion and ultrasmall stomatocytes. Scale bar: $(a-c) 500 \mathrm{~nm}$; $\left(a_{1}-\right.$ $\left.c_{1}\right) 100 \mathrm{~nm}$.

MPS clearance and longer circulation time. ${ }^{16}$ Hence, developing delivery vehicles smaller than $200 \mathrm{~nm}$ with the capability of longer circulation time and tumor vasculature penetration is still challenging.

Our group has previously used a "bottom-up approach" to fabricate nanomotors by changing the shape of supramolecular assembled polymersomes into bowl-shaped stomatocytes. ${ }^{26}$ These stomatocyte nanomotors have been fabricated to have unique properties such as controlled drug release, ${ }^{27}$ controlled speed, ${ }^{28}$ and protein encapsulation. ${ }^{29}$ Although the size of our stomatocyte motors is at the nanoscale, they are still larger than $200 \mathrm{~nm}$, which is not ideal for systemic delivery and biomedical applications. Moreover, the method we developed for protein encapsulation is laborious, time-consuming, and not achievable with smaller-sized particles.
In this paper, we have designed ultrasmall stomatocyte nanomotors (USSNs) of around $150 \mathrm{~nm}$ by using the extrusion method. In our previous work, several methodologies designed to achieve the structural transformation of polymersomes into stomatocytes have been developed, such as direct dialysis, $^{30}$ reverse dialysis, and organic solvent addition. ${ }^{26,29}$ However, none of these methods were suitable to transform smaller-sized polymersomes into stomatocytes. ${ }^{27,31-35}$ This may be due to the osmotic pressure created by these methods, which is not high enough to cause the shape transformation of smaller polymersomes. Recently, a new methodology for tuning the shape of polymersomes has been described, which involves adding PEG to the flexible polymersomes, where PEG is considered to create a higher osmotic shock for shape transformation. ${ }^{36}$ Here, PEG solution was added to the 
extruded solution in the presence of enzymes (to power motion via chemical gradients) to form uniform USSNs. By using this method, we can encapsulate proteins and thus protect them from denaturation. These size-controlled stomatocytes are also reversible, meaning that they can be converted back to polymersomes by adding organic solvent (Figure 1). Moreover, the USSN studied in this way was more efficient at extravasating the tumor vasculature system and accumulating into tumor cells in comparison to previously reported stomatocyte nanomotors.

\section{RESULTS AND DISCUSSION}

Self-Assembly of Stomatocytes with Controlled Size. Polymersomes were fabricated by self-assembly of diblock copolymers with an average size of $371.1 \mathrm{~nm}$ (Figure $2 \mathrm{~d}$ ). The polymersomes thus formed were extruded with a filter membrane with a pore size of $220 \mathrm{~nm}$ to form ultrasmall polymersomes of around $150.3 \mathrm{~nm}$ (Figure 2d). The size and morphology of both polymersomes were studied using dynamic light scattering (DLS), transmission electron microscopy (TEM), and cryo-TEM (Figure 2a,b,d). After uniform ultrasmall polymersomes were formed, PEG solution was added to provide an instantaneous osmotic shock for the shape transformation from polymersomes to stomatocytes. ${ }^{36}$ Compared to our previously synthesized stomatocytes, more PEG was required for shape transformation of ultrasmall polymersomes as smaller vesicles with higher curvature stress need more osmotic pressure for membrane folding. ${ }^{37}$ The synthesized ultrasmall stomatocytes were around $153.6 \mathrm{~nm}$ and were very uniform with a small opening as observed by DLS, TEM, and cryo-TEM (Figure 2c,d). As has previously been shown, osmotic pressure induced by the presence of additives in the solution causes shape transformation because the inside volume of polymersomes will decrease according to the different osmotic pressure created by different amounts of molecules introduced. ${ }^{37-39}$ In our study, the PEG solution circumvents the limitation in the osmotic shock of other methods such as dialysis and the addition of organic solvents and induced the shape changing of small polymersomes, thus allowing the fabrication of ultrasmall stomatocyte nanomotors.

Effects of Amphiphile Molecular Weight and Bilayer Thickness on Stomatocyte Formation. Additive PEG addition methodology is suitable for fabricating stomatocyte polymersomes even in the ultrasmall size scale; however, whether this system is robust and can be systematically used for polymersomes within different parameters is still not clear. For example, molecular weight is an important factor for forming polymersomes and might influence the bilayer's thickness. In order to investigate this effect and test the suitability of the technique to a wider range of amphiphiles, we performed the shape transformation using the PEG addition method on polymersomes assembled from amphiphiles with different units of $\mathrm{PS}_{n}$. Briefly, $\mathrm{PEG}_{44}-b-\mathrm{PS}_{n}$ block copolymers were assembled as described previously into polymersomes, which were further extruded to produce small polymersomes with different membrane thicknesses. With the addition of PEG solution, polymersomes reshaped into stomatocytes. As shown in Figure 3, for polymersomes formed from amphiphiles with 170 units of PS, $5 \mu \mathrm{L}$ of PEG solution led to a mixture of prolates, tubes, and stomatocytes with a large opening, whereas with $10 \mu \mathrm{L}$ of PEG solution, a small opening of stomatocytes was found, and the ratio of small opening stomatocytes increases to $100 \%$ when $20 \mu \mathrm{L}$ of PEG solution was added
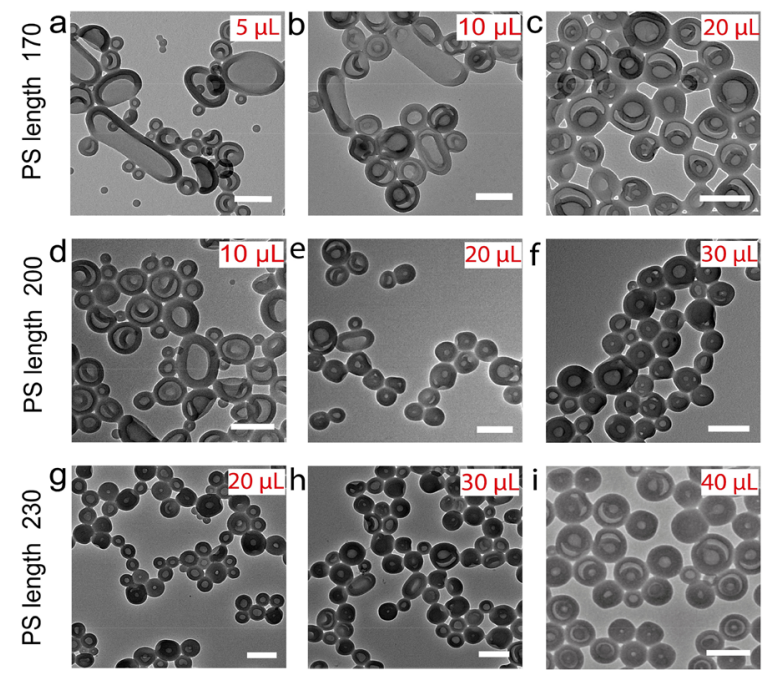

Figure 3. TEM for shape transformation of small polymersomes into small stomatocytes, with different PS lengths and different amounts of PEG2000. ( $a-c)$ PS length of 170, with the addition of 5, 10, and $20 \mu \mathrm{L}$ of $100 \mathrm{mg} / \mathrm{mL}$ PEG solution; (d-f) PS length of 200, with the addition of 10, 20, and $30 \mu \mathrm{L}$ of $100 \mathrm{mg} / \mathrm{mL}$ PEG solution; $(g-i)$ PS length of 230 , with the addition of 20,30 , and $40 \mu \mathrm{L}$ of $100 \mathrm{mg} / \mathrm{mL}$ PEG solution. Scale bars: $200 \mathrm{~nm}$.

(Figure $3 a-c)$. For the 200 and 230 units of PS polymersomes, the shape transformation trends are similar to those of 170 units of polymersomes, and it is worth noting that the amount of PEG solution was also increased within the increasing of PS units. Therefore, 30 and $40 \mu \mathrm{L}$ of PEG solution was required to fabricate pure small opening stomatocytes instead of $20 \mu \mathrm{L}$ of PEG solution (Figure 3d-i). Theoretically, molecular weight of amphiphiles affects the thickness of bilayers as $d \sim$ $\left(M_{\mathrm{w}}\right)^{0.5}$ ( $d$ is the core thickness), and it is also supported by cryo-TEM measurements. ${ }^{40}$ This has been indeed confirmed by electron microscopy measurements, as shown in Figure S1; a membrane thickness of 170, 200, and 230 units of PS stomatocyte polymersomes was measured and found to be around 15, 17.5, and $20 \mathrm{~nm}$, respectively. The difference in membrane thickness causes the differences in curvature stress and the bending energy required for shape transformation. ${ }^{39}$ The PEG addition method is therefore promising for shape transformation of different polymers, and the introduced osmotic pressure is enough for bending the bilayers, even though PS units were increased to 230, as long as the amount of PEG was also optimized.

Encapsulation of Catalase. Enzyme-powered motors have been widely studied as promising new generation motors as enzymes are considered to be versatile and biocompatible alternative catalysts and also show higher efficiency for propelling motors, which makes enzyme-powered motors ideal tools for medical research. ${ }^{41,42}$ Several enzymes have been used as biocatalysts, such as urease, catalase, glucose oxidase, alkaline phosphatase, etc., ${ }^{41,43}$ which inspired us to fabricate ultrasmall nanomotors with a biocatalyst system. To fabricate USSN, catalase was encapsulated in the inner compartments of stomatocytes. This was carried out by adding catalase to the extruded polymersomes followed by the PEG addition to the solution. During the shape transformation of polymersomes, catalase is encapsulated in the stomach of the 

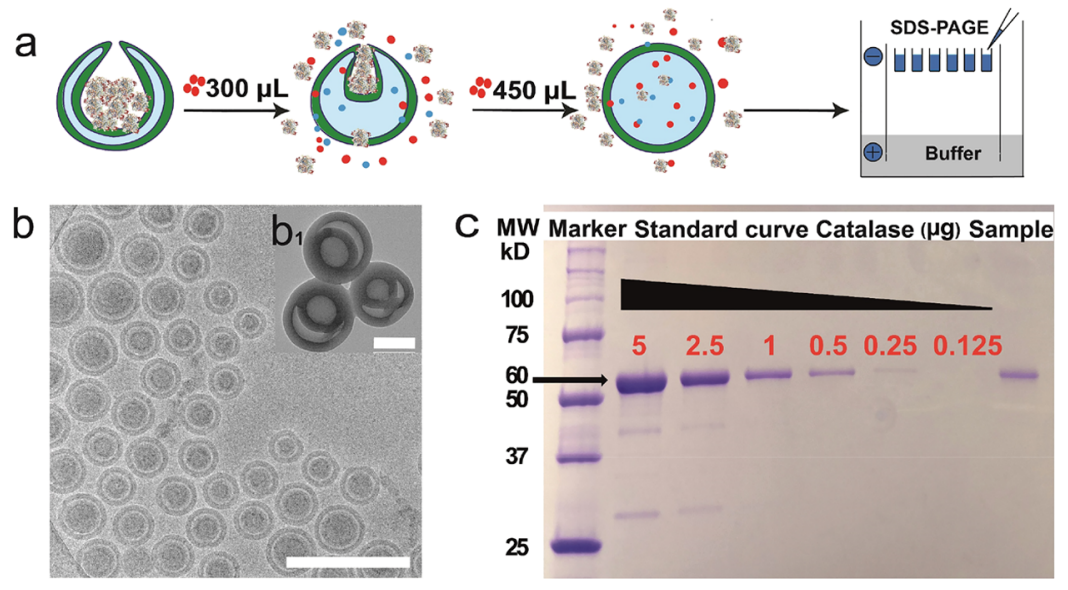

Figure 4. (a) Scheme for the study of encapsulation efficiency. TEM (b) and cryo-TEM images ( $\left.b_{1}\right)$ of size-controlled stomatocytes encapsulated with enzymes. (c) SDS-PAGE of released enzymes from the stomatocytes and from a catalase standard curve solution. Scale bar: (b) $500 \mathrm{~nm}$; $\left(b_{1}\right) 100 \mathrm{~nm}$.
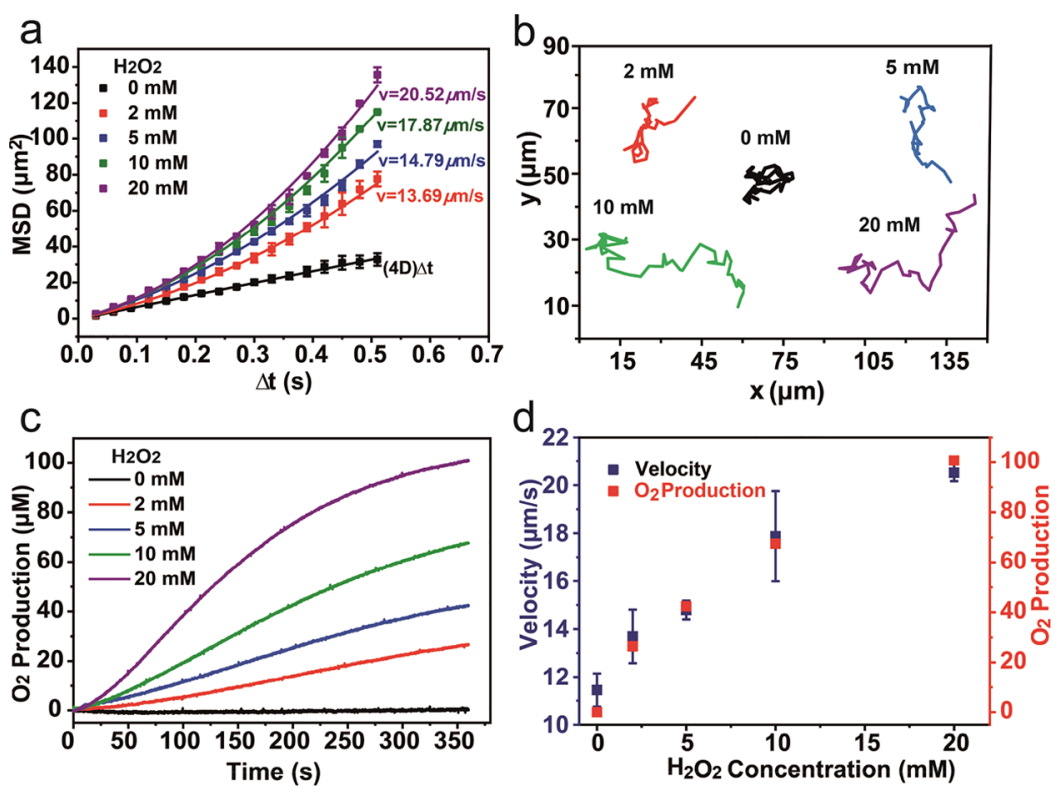

Figure 5. Movement analysis of catalase-driven ultrasmall stomatocyte nanomotors at different concentrations $\left(2-20 \mathrm{mM} \mathrm{H}_{2} \mathrm{O}_{2}\right)$. (a) Velocity of catalase-encapsulated ultrasmall stomatocyte nanomotors at different fuel concentrations; the velocity was extracted from the fitting of the average MSD of catalase-encapsulated ultrasmall stomatocyte, calculated from the tracking coordinates of, on average, 50 particles. (b) Typical tracking paths of nanomotors, recorded for 2 s. (c) Oxygen production of catalase-encapsulated ultrasmall stomatocyte nanomotors. (d) Relation between velocity and oxygen production.

stomatocytes, which allows the assembly of the nanomotor propelling the structures in the presence of $\mathrm{H}_{2} \mathrm{O}_{2}$. TEM and cryo-TEM were used to visualize the morphology of these samples (Figure $\left.4 \mathrm{~b}-\mathrm{b}_{1}\right)$. More PEG was required $(50 \mu \mathrm{L})$ to achieve the shape transformation of polymersomes in the presence of catalase in comparison to the transformation without catalase in the reaction mixture. The increase of PEG required for the shape transformation might be due to the interaction between enzymes and the membrane of polymersomes; however, no significant change in the final morphology of stomatocytes was observed.

Releasing of the encapsulated cargoes from the stomach of stomatocytes is also important for further studies, which will give us more information on the encapsulation efficiency of biologics, etc. Whether rigid stomatocytes can turn back to polymersomes or not therefore becomes pivotal. As shown in
Figure S2, when the starting point of the shape transformation changed to rigid stomatocytes with enzymes encapsulated, the reversibility of USSN was observed by slowly adding organic solvent. From TEM and cryo-TEM images, the reverse shape transformation was initiated with $150 \mu \mathrm{L}$ of organic solvent to stomatocyte solution and is completed when the organic solvent addition reaches $450 \mu \mathrm{L}$, after which most of the stomatocytes change back to polymersomes. After releasing catalase from the stomach, the encapsulation efficiency was quantified by SDS-PAGE. Figure $4 \mathrm{c}$ showed the standard curve of catalase, which was used to quantify the amount of catalase released from stomatocytes. The correlation curves and the quantified bands are shown in Figure S3. Amplex red assay was employed to study the activity of the encapsulated catalase, and the calibration curve for enzyme activity is shown in Figure S3c. Based on these curves, $16.53 \mu \mathrm{g}$ of catalase was 
determined to be encapsulated via SDS-PAGE, which is different from the result from the activity assay $(12.47 \mu \mathrm{g})$; the discrepancy is most probably due to the denaturation of proteins during the loading procedure. After the encapsulation, enzyme activity decreased by $24.57 \%$. Compared to previous research with nanoporous silica spheres that retained $62 \%$ activity of the encapsulated enzyme, ${ }^{44}$ this method kept enzymes more active. In our previous described methods for enzyme encapsulation, enzymes were exposed to plasticizers for $30 \mathrm{~min},{ }^{29}$ which might affect even further their activity. By using this method for encapsulating proteins, they would just be exposed to organic solvent for several seconds, which protects the enzyme from denaturation. Moreover, the existence of PEG solution plays the role of additive in the stabilization of proteins. ${ }^{45,46}$ Thus, by selecting the appropriate encapsulation method, we were able to stabilize the enzyme and preserve its activity quite well for further analysis of movement for biomedical applications. In terms of protein encapsulation, this method can also be applied systematically. With the polymeric coatings, proteins can be protected from immunogenicity, which is promising for protein therapeutics. $^{47,48}$

Motion Characterization of Ultrasmall Stomatocyte Nanomotors. To study the autonomous movement of enzyme-powered USSNs, nanoparticle tracking analysis was carried out to record and analyze the real-time movement of particles. ${ }^{26}$ USSNs without fuel (controls) displayed Brownian motion, which was confirmed by the linear fitting of the MSD curve $\left(\left\langle r^{2}\right\rangle=4 D t\right) .{ }^{29,49}$ Adding different concentrations of $\mathrm{H}_{2} \mathrm{O}_{2}$ to the catalase-encapsulated USSN solution resulted in a parabolic fitting of MSD curves (Figure 5a). The velocity of USSN increased from $13.69 \pm 1.11$ to $20.52 \pm 0.35 \mu \mathrm{m} / \mathrm{s}$ with an increase in concentration of $\mathrm{H}_{2} \mathrm{O}_{2}$ from 2 to $20 \mathrm{mM}$. The USSN measured speeds of 117 body lengths/s in the presence of $10 \mathrm{mM} \mathrm{H}_{2} \mathrm{O}_{2}$, which is quite fast when compared to other motors of similar size. ${ }^{50}$ Additionally, these motors have a smaller size that could explain the higher speeds as less energy is required to power smaller particles. ${ }^{29}$ Research from other groups also tried to lower the concentration of $\mathrm{H}_{2} \mathrm{O}_{2}$, for example, $0.5 \% \mathrm{H}_{2} \mathrm{O}_{2}{ }^{51}$ and $1.5 \mathrm{wt} \%{ }^{52}$ were used for powering motors, which are still higher than $20 \mathrm{mM}(0.06 \%)$. The sizes of these motors are still at the microscale, which made them more difficult to be propelled. However, with similar-sized Janus particles, speeds lower than those with our stomatocyte nanomotors were recorded, which could be attributed to the catalyst choice; in this case, Pt nanoparticles were used for the Janus system which has catalytic efficiency lower than that of catalase, showcasing the importance of catalyst choice used to power the motion as well as the shape of the nanomotors. ${ }^{50,53}$ Sample trajectories for the movement of nanomotors at different $\mathrm{H}_{2} \mathrm{O}_{2}$ concentration is shown in Figure $5 \mathrm{~b}$. At $0 \mathrm{mM}$, only Brownian motion was observed with no net displacement of the particles. However, an increase in net displacement was observed with an increase in concentration of fuel. The mechanism of motion for bubble-propelled motors has been extensively studied; basically the velocity of motors is mainly dependent on the consumption of $\mathrm{H}_{2} \mathrm{O}_{2}$ and the $\mathrm{O}_{2}$ production and the formation of bubbles. ${ }^{54-56}$ However, most of these studies were based on tubular motors and also on a microscale, which may not provide direct evidence for further understanding of nanoscale bubble-propelled motors. On the other hand, tracking nanoscale motors is still a challenge as they are too small to be observed using optical microscopy techniques, let alone see the bubble production. By using an oxygraph system, the oxygen produced by decomposing $\mathrm{H}_{2} \mathrm{O}_{2}$ can be detected, which may give us some information on the relationship between bubble production and the movement of nanomotors. Here, to demonstrate that the movement of USSNs is related to the decomposition of $\mathrm{H}_{2} \mathrm{O}_{2}$, the $\mathrm{O}_{2}$ evolution rate was studied for different $\mathrm{H}_{2} \mathrm{O}_{2}$ concentrations (Figure 5c). As expected, increased oxygen evolution was observed with an increase in $\mathrm{H}_{2} \mathrm{O}_{2}$ concentration, and this directly correlated with the increase in the speed of the nanomotors (Figure 5d), which suggested that bubble production might be the essential factor in powering motors.

Enhanced Penetration Across Vasculature Models and Internalization of Nanomotors with Controlled

Size. To accumulate nanoparticles into tumor tissues, a passive delivery pathway based on the enhanced permeation and retention (EPR) effect has been actively used, due to the presence of immature and permeable tumor vasculature. ${ }^{57-59}$ However, this passive method can also be limited by the pore size of these leaky vessels. Furthermore, most of the tumors have a vascular pore size ranging from 100 to $800 \mathrm{~nm},{ }^{21,60,61}$ and nanoparticles with a size of around $130 \mathrm{~nm}$ produced promising results from the perspective of the EPR effect. ${ }^{62}$ In this study, the penetration of nanomotors through a confluent monolayer of pulmonary artery endothelial cells was studied to investigate the size influence of nanomotors when passing through the vasculature model. Corning transwell inserts with porous membranes (pore size $3 \mu \mathrm{m}$ ) were used to seed cells, and small stomatocyte nanomotors (SSNs) and USSNs made by a PEG addition method (Figure S4) were added on the top compartments of the insets, as shown in Figure S5a. Nanomotors in the top and bottom compartments were collected and washed by centrifugation after $2 \mathrm{~h}$ incubation. Fluorescence intensity was studied using a plate reader. As shown in Figure S5b, in comparison with SSN particles, USSN particles' translocation increased by $13.64 \%$, from 38.59 to $52.23 \%$ in the absence of $\mathrm{H}_{2} \mathrm{O}_{2}$. When $2 \mathrm{mM} \mathrm{H}_{2} \mathrm{O}_{2}$ was added as a fuel for the motion of nanomotors, $65.93 \%$ of USSN particles were present in the bottom chamber, compared to SSN particles (48.91\%). A $17 \%$ increase was observed, showing that USSN is much better for undergoing tumor vasculature both with and without fuel. To demonstrate the formation of EPR model, transepithelial/transendothelial electrical resistance (TEER) was measured via a chopstick electrode EVOM2 (World Precision Instruments). The TEER was also measured after conducting the experiment, to study whether the cell monolayer permeability was changed by the motors and the addition of $\mathrm{H}_{2} \mathrm{O}_{2}$. The morphology of the monolayer endothelial cells was also studied by confocal laser scanning microscopy (CLSM) before and after the experiment. As shown in Figure S6, the cell monolayer was formed, and no difference was found from the TEER and cell morphology studies before and after the experiment was conducted, as incubation time is limited.

It is also well-known that cellular uptake of nanoparticles (for example, liposomes, ${ }^{63}$ gold particles, $^{64}$ and silica particles ${ }^{65}$ ) is size-dependent and plays a major role in the uptake efficiency and endocytic pathway, although physicochemical properties such as surface charge and surface functionality could also affect the internalization of NPs. ${ }^{17}$ When it comes to motors, similar rules are expected to apply, although the scenario is more complex because factors like 

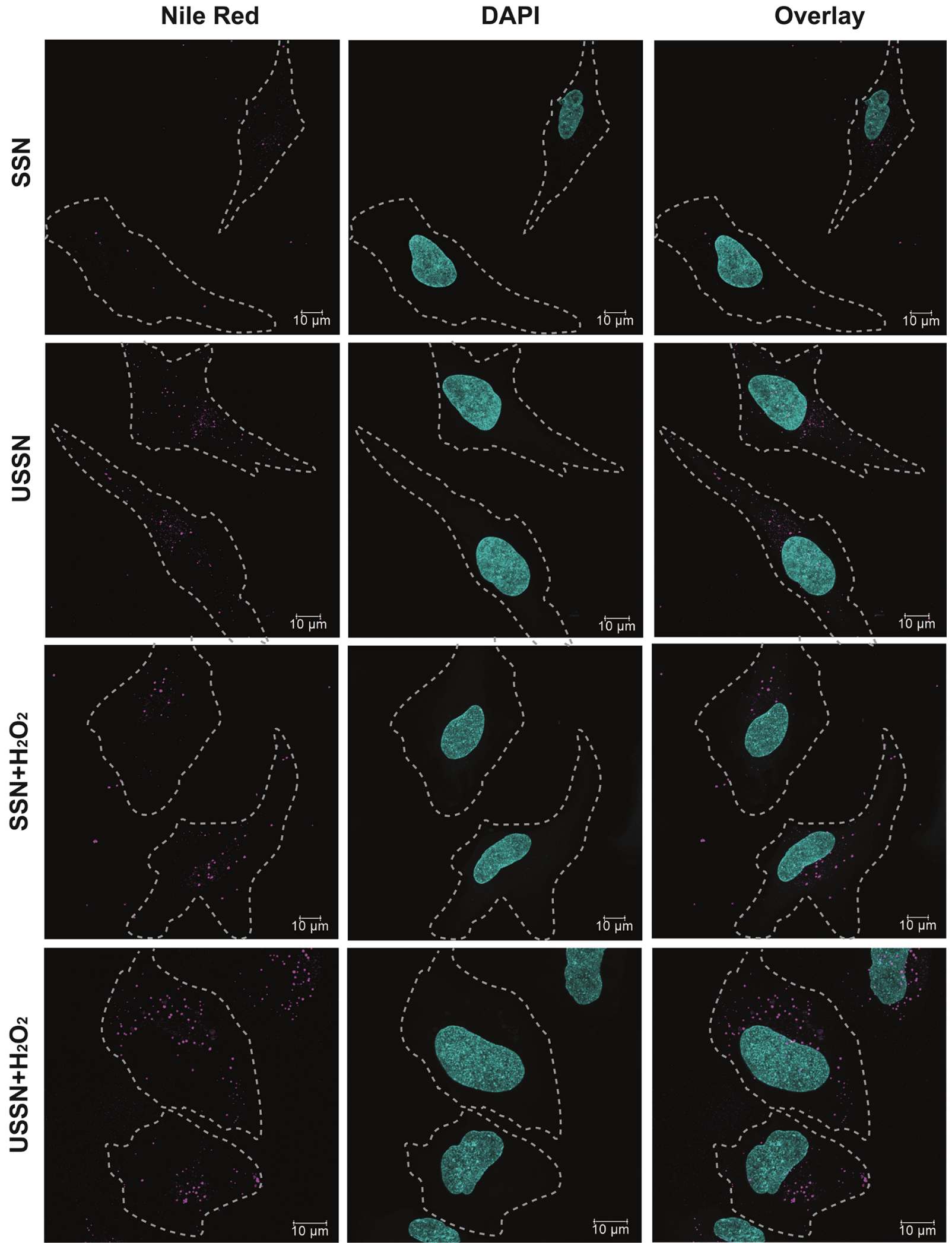

Figure 6. HeLa cells incubated with nanomotors and ultrasmall nanomotors in the presence of $2 \mathrm{mM} \mathrm{H}_{2} \mathrm{O}_{2}$ and without hydrogen peroxide for $3.5 \mathrm{~h}$. Cell nuclei were dyed with DAPI after cells were fixed; Nile Red was encapsulated in the nanomotors for fluorescence. Scale bar: $10 \mu \mathrm{m}$.

interactions between active motors and dynamic surfaces (such as cell membranes), fuels that are used to power the motors and mechanism of motion, can affect particle uptake. In this experiment, we compared two nanomotors with different size distributions fabricated by the same polymer to investigate the influence of size on cellular uptake using human cervical cancer cells (HeLa cells) as a model cell. The material toxicity was studied by detecting the cell viability after the nanomotors 
were incubated with HeLa cells for $24 \mathrm{~h}$. As shown in Figure S8, no significant cytotoxicity was observed after the cells were incubated with ultrasmall stomatocyte polymersomes and ultrasmall stomatocyte nanomotors. Similar results were also observed when cells were incubated for a short time scale (3.5 h) and studied by CLSM, as shown in Figure S9, in which the cell viability was detected by propidium iodide. A Leica Microsystems TSC SP8 HyVolution confocal microscope was used to visualize the cellular uptake after HeLa cells were incubated with SSN and USSN for $3.5 \mathrm{~h},{ }^{66}$ and the fluorescence area from Nile Red was quantified with Fiji. As shown in Figure 6, the USSN showed a cellular uptake efficiency higher than that of the SSN, with 3.7 times higher fluorescence area observed. Although in the presence of $2 \mathrm{mM}$ $\mathrm{H}_{2} \mathrm{O}_{2}$, USSN showed higher uptake efficiency and the fluorescence area from Nile Red was 2.2 times more than that of the SSN. Compared to the samples without $\mathrm{H}_{2} \mathrm{O}_{2}$, there was increased internalization with both nanomotors, which is mostly due to the active movement of motors as no cell death was observed during the experiment. 3D videos of these cells were made to prove that nanomotors were actually embedded inside the cells instead of attaching to the membrane (Video S1 and Video S2). Moreover, flow cytometry was also recruited for further uptake quantification. As shown in Figure S11, USSNs showed the highest uptake efficiency with the presence of fuel, following the trend observed by CLSM. To study whether $\mathrm{H}_{2} \mathrm{O}_{2}$ damaged the cell membrane during the experiment, which might lead to a higher uptake but not the motion of nanomotors, a cell membrane permeability assay was conducted using propidium iodide because it is not membrane-permeable. As shown in Figure $\mathrm{S} 10, \mathrm{H}_{2} \mathrm{O}_{2}$ had almost no influence on the cells when nanomotors were added to incubate with the cells. In contrast, when polymersomes were added together with $\mathrm{H}_{2} \mathrm{O}_{2}$, the cell membrane was more permeable. The difference between samples was the presence of catalase that decomposes $\mathrm{H}_{2} \mathrm{O}_{2}$ to $\mathrm{O}_{2}$, which decreases the $\mathrm{H}_{2} \mathrm{O}_{2}$-related damage. As the $\mathrm{H}_{2} \mathrm{O}_{2}$ concentration decreases, moreover, catalase has also been used as an antioxidant enzyme to detoxify $\mathrm{H}_{2} \mathrm{O}_{2}$, ${ }^{67}$ which protects cells from being damaged. The above results suggest that smaller-sized nanomotors are more promising for biomedical applications, as they exhibit positive results in passing through the leaking vessels and cell membranes.

\section{CONCLUSIONS}

In summary, we have successfully fabricated ultrasmall stomatocyte polymersomes with uniform size $(150 \mathrm{~nm})$ by applying the PEG addition method, and we have demonstrated that this method can be systematically used for the shape transformation of polymersomes under different conditions. Biocompatible catalyst catalase was encapsulated inside these ultrasmall stomatocyte nanomotors in several seconds. By decomposing $\mathrm{H}_{2} \mathrm{O}_{2}, \mathrm{O}_{2}$ was produced to power the motion of USSNs even with a very low concentration of fuel. Furthermore, the moving velocity of USSNs was found to have a positive correlation with $\mathrm{O}_{2}$ production, which is also concentration-dependent at the same time. The capability of encapsulating enzymes in the inner compartment makes the USSN a promising nanovesicle for biomedical applications, such as protein delivery and imaging. Compared to small stomatocyte nanomotors, USSNs were more efficient in penetrating a confluent monolayer of pulmonary artery endothelial cells, which suggests that more cargoes can be delivered by USSNs than SSNs through the EPR effect. Moreover, USSNs were much more capable of internalizing into the HeLa cells with or without the presence of $\mathrm{H}_{2} \mathrm{O}_{2}$ due to the smaller size range and faster motion.

\section{MATERIALS AND METHODS}

All reagents and chemicals were purchased from commercial sources and used as received. Milli- $Q$ water $(18.2 \mathrm{M} \Omega)$ was used in all of the experiments. Catalase (E3289) from bovine liver, $\geq 20000$ units/mg protein, lyophilized powder, was purchased from Sigma-Aldrich.

Dynamic light scattering experiments were studied on a Malvern Zetasizer Nano S equipped with a $\mathrm{He}-\mathrm{Ne}(633 \mathrm{~nm}, 4 \mathrm{~mW})$ laser and an Avalanche photodiode detector at an angle of $173^{\circ}$. Transmission electron microscopy samples were prepared in the following way: a solution of sample $(5 \mu \mathrm{L})$ was air-dried on a carbon-coated Cu TEM grid (200 mesh). A JEOL TEM 1400 microscope at an acceleration voltage of $120 \mathrm{kV}$ and a JEOL TEM 2100 at an acceleration voltage of $200 \mathrm{kV}$ were used for the characterization of shape transformation.

Synthesis of Poly(ethylene glycol) ${ }_{44}-b$-Poly(styrene $)_{n}$. Polymerization of PEG-b-PS was reported in previous research. ${ }^{30}$ Molecular weights of the block copolymers were measured on a Shimadzu Prominence GPC system equipped with a PL gel $5 \mu \mathrm{m}$ mixed D column (Polymer Laboratories) and differential refractive index and UV (254 nm) detectors. NMR spectra were obtained on a Varian Inova 400 spectrometer in $\mathrm{CDCl}_{3}$.

Preparation of Polymersomes. Modified from our previous research, ${ }^{36} \mathrm{PEG}_{44}-b$ - $\mathrm{PS}_{n}(10 \mathrm{mg})$ was dissolved in $1 \mathrm{~mL}$ of an organic solvent mixture of tetrahydrofuran (THF) and 1,4-dioxane in a 4:1 ratio by volume with a magnetic stirring bar. After the solution was dissolved for $30 \mathrm{~min}, 0.5 \mathrm{~mL}$ of Milli-Q water was added to the solution at a rate of $1 \mathrm{~mL} \mathrm{~h}^{-1}$ at room temperature with vigorous stirring $(900 \mathrm{rpm})$ to make polymersomes. Ultrasmall polymersomes were fabricated by passing $700 \mu \mathrm{L}$ of polymersome solution through a $220 \mathrm{~nm}$ syringe filter (screening devices) in a mixture solvent of $\mathrm{THF} /$ dioxane $/$ water $=8 / 2 / 5$ by volume .

Preparation of Ultrasmall Stomatocytes and Encapsulation of Enzymes Inside the Stomatocyte Cavity via the PEG Addition Method. The procedures for preparation of polymersomes were the same as those described above. After polymersomes were made, $30 \mu \mathrm{L}$ of PEG2000 aqueous solution $(100 \mathrm{mg} / \mathrm{mL})$ was added into the ultrasmall polymersome suspension $(550 \mu \mathrm{L})$ under a stirring speed of $900 \mathrm{rpm}$ for $1 \mathrm{~min}$. Ten milliliters of Milli-Q water was poured into the solution to quench the structure. For encapsulating enzymes inside stomatocytes, $1 \mathrm{mg}$ of catalase was dissolved in $50 \mu \mathrm{L}$ of water and was added into the polymersome solution or ultrasmall polymersome solution. After being mixed for 2 min with a stirring plate, an appropriate amount of PEG2000 solution $(100 \mathrm{mg} / \mathrm{mL})$ was added and the solutions were quenched as described. The organic solvent and non-encapsulated enzymes in the system were removed by centrifugation.

Solvent Addition Method for Reshaping Stomatocytes. First, $500 \mu \mathrm{L}$ of a rigid USSN solution was transferred into a $5 \mathrm{~mL}$ vial. A THF/dioxane $(4: 1 \mathrm{v} / \mathrm{v})$ mixture was added via a syringe pump with the rate of $300 \mu \mathrm{L} \mathrm{h}^{-1}$ under a stirring speed of $900 \mathrm{rpm}$ by using a stirring plate. Samples were withdrawn and quenched at different time points $(30,60,90$, and $180 \mathrm{~min})$. TEM and cryo-TEM sample were made to follow the shape transformation.

Release of Enzymes from the Stomatocyte Cavity. Encapsulated catalase was released from the stomatocyte by the solvent addition method: $450 \mu \mathrm{L}$ of THF/dioxane $(4: 1 \mathrm{v} / \mathrm{v})$ was added to $500 \mu \mathrm{L}$ of rigid USSN solution as described above to reshape the stomatocytes. Five milliliters of Milli-Q water was added to quench the structure and dilute the organic solvent. Spin filters with a $10 \mathrm{kDa}$ membrane was used to remove the organic solvent and collect the released enzymes. The presence of free enzymes was checked by SDS-PAGE.

SDS-PAGE Analysis. SDS-PAGE was performed on a minivertical electrophoresis system (Bio-Rad Mini-PROTEAN 2 Cell, Bio$\mathrm{Rad}$ Laboratories). Dilutions of catalase ranging from $5 \mu \mathrm{g}$ to $125 \mathrm{ng}$ 
were run on $10 \%$ Tris-glycine precast gels at $120 \mathrm{~V}$ for $60 \mathrm{~min}$. An electrode buffer ( $0.1 \%$ SDS, $0.25 \mathrm{M}$ glycine, $0.025 \mathrm{M}$ Tris- $\mathrm{HCl}$, $\mathrm{pH}$ 8.3) was used as described in the manufacturer's instructions. The gels were stained by Coomassie Brilliant Blue R-250 staining solution and washed by destaining solution for several times.

Autonomous Movement of the Stomatocyte Nanomotors. The movement of nanomotors was evaluated at five different $\mathrm{H}_{2} \mathrm{O}_{2}$ concentrations $(0,2,5,10$, and $20 \mathrm{mM}$ ). Nanomotors (final concentration around $3 \times 10^{8}$ particles $\mathrm{mL}^{-1}$ ) were quickly injected into the NanoSight LM10 sample chamber after being mixed with $\mathrm{H}_{2} \mathrm{O}_{2}$ solution. Videos (each video duration time $30 \mathrm{~s}$, rate 30 frames $\mathrm{s}^{-1}$ ) were recorded, and the particle movement was analyzed with nanoparticle tracking software (NTA 2.2).

Cell Uptake of Stomatocyte Nanomotors. Two hundred microliters of Dulbecco's modified Eagle's medium (DMEM) buffer with $1 \times 10^{5}$ cells was added to an 8-well plate (ibidi GmbH) and incubated overnight at $37{ }^{\circ} \mathrm{C}$. The incubation buffer was then replaced by $200 \mu \mathrm{L}$ of DMEM buffer with stomatocyte nanomotors (particle concentration $=2 \times 10^{10}$ particles $/ \mathrm{mL}$ ). After $3.5 \mathrm{~h}$ incubation with or without $2 \mathrm{mmol} \mathrm{H}_{2} \mathrm{O}_{2}$, the cell culture medium was removed, and cells were washed with PBS three times and fixed with $4 \%$ paraformaldehyde in PBS for $10 \mathrm{~min}$ at room temperature. For nuclear staining, 4',6-diamidino-2-phenylindole (DAPI) (Millipore) was added and incubated for $1 \mathrm{~h}$. After the staining solution was removed, cells were washed with PBS buffer three times. The stained cells were imaged by SP $8 \times$ confocal microscope, and the presence of fluorescence was measured by Fiji.

\section{ASSOCIATED CONTENT}

\section{(5) Supporting Information}

The Supporting Information is available free of charge on the ACS Publications website at DOI: 10.1021/acsnano.9b03358.

Materials, experimental section, TEM and cryo-TEM images, calibration curves, DLS, schematics (PDF)

Video $\mathrm{S} 1$ shows the 3D structure of cell uptake for USSN with $\mathrm{H}_{2} \mathrm{O}_{2}$ (AVI)

Video S2 shows the 3D structure of cell uptake for USSN without $\mathrm{H}_{2} \mathrm{O}_{2}$ (AVI)

\section{AUTHOR INFORMATION}

\section{Corresponding Author}

*E-mail: d.wilson@science.ru.nl.

\section{ORCID}

Daniela A. Wilson: 0000-0002-8796-2274

\section{Author Contributions}

J.S. and D.A.W. designed the experiments. J.S. performed the experiments and analyzed the results. J.S., M.M., and W.L. interpreted the results. J.S., M.M., and D.A.W. wrote the manuscript.

\section{Notes}

The authors declare no competing financial interest.

\section{ACKNOWLEDGMENTS}

The authors would like to thank G.-J. Janssen and I. Alexopoulos from General Instruments at Radboud University for assistance with the cryo-TEM analysis and confocal images. D.A.W. acknowledges the NWO Chemiche Wetenschappen VIDI Grant No. 723.015.001 for financial support. We acknowledge support from the Ministry of Education, Culture and Science (Gravity Program 024.001.035). M.M. would like to thank European Union's Horizon 2020 framework program for funding under the Marie Skłodowska-Curie Individual Fellowships Grant Agreement No. 794657.

\section{REFERENCES}

(1) Cross, R. L. Turning the ATP Motor. Nature 2004, 427, 407.

(2) Hirokawa, N.; Noda, Y.; Tanaka, Y.; Niwa, S. Kinesin Superfamily Motor Proteins and Intracellular Transport. Nat. Rev. Mol. Cell Biol. 2009, 10, 682.

(3) Palagi, S.; Fischer, P. Bioinspired Microrobots. Nat. Rev. Mater. 2018, 3, 113

(4) Paxton, W. F.; Kistler, K. C.; Olmeda, C. C.; Sen, A.; St. Angelo, S. K.; Cao, Y.; Mallouk, T. E.; Lammert, P. E.; Crespi, V. H. Catalytic Nanomotors: Autonomous Movement of Striped Nanorods. J. Am. Chem. Soc. 2004, 126, 13424-13431.

(5) Ismagilov, R. F.; Schwartz, A.; Bowden, N.; Whitesides, G. M. Autonomous Movement and Self-Assembly. Angew. Chem., Int. Ed. 2002, 41, 652-654.

(6) Soong, R. K.; Bachand, G. D.; Neves, H. P.; Olkhovets, A. G.; Craighead, H. G.; Montemagno, C. D. Powering an Inorganic Nanodevice with a Biomolecular Motor. Science 2000, 290, 15551558.

(7) Gao, W.; Wang, J. The Environmental Impact of Micro/ Nanomachines: A Review. ACS Nano 2014, 8, 3170-3180.

(8) Tu, Y.; Peng, F.; Wilson, D. A. Motion Manipulation of Microand Nanomotors. Adv. Mater. 2017, 29, 1701970.

(9) Dey, K. K.; Wong, F.; Altemose, A.; Sen, A. Catalytic MotorsQuo Vadimus? Curr. Opin. Colloid Interface Sci. 2016, 21, 4-13.

(10) Palagi, S.; Walker, D.; Qiu, T.; Fischer, P. Nanoscale Robotic Agents in Biological Fluids and Tissues. In The Encyclopedia of Medical Robotics; World Scientific: Singapore, 2018; pp 19-42.

(11) Xi, W.; Solovev, A. A.; Ananth, A. N.; Gracias, D. H.; Sanchez, S.; Schmidt, O. G. Rolled-up Magnetic Microdrillers: Towards Remotely Controlled Minimally Invasive Surgery. Nanoscale 2013, 5, 1294-1297.

(12) Balasubramanian, S.; Kagan, D.; Jack Hu, C. M.; Campuzano, S.; Lobo-Castañon, M. J.; Lim, N.; Kang, D. Y.; Zimmerman, M.; Zhang, L.; Wang, J. Micromachine-Enabled Capture and Isolation of Cancer Cells in Complex Media. Angew. Chem. 2011, 123, 42474250.

(13) Wu, Z.; Li, J.; de Ávila, B. E. F.; Li, T.; Gao, W.; He, Q.; Zhang, L.; Wang, J. Water-Powered Cell-Mimicking Janus Micromotor. Adv. Funct. Mater. 2015, 25, 7497-7501.

(14) Wu, Z.; Wu, Y.; He, W.; Lin, X.; Sun, J.; He, Q. Self-Propelled Polymer-Based Multilayer Nanorockets for Transportation and Drug Release. Angew. Chem., Int. Ed. 2013, 52, 7000-7003.

(15) Peng, F.; Tu, Y. F.; Adhikari, A.; Hintzen, J. C. J.; Lowik, D. W. P. M.; Wilson, D. A. A Peptide Functionalized Nanomotor as an Efficient Cell Penetrating Tool. Chem. Commun. 2017, 53, 10881091.

(16) Elsabahy, M.; Wooley, K. L. Design of Polymeric Nanoparticles for Biomedical Delivery Applications. Chem. Soc. Rev. 2012, 41, $2545-2561$

(17) Iversen, T. G.; Skotland, T.; Sandvig, K. Endocytosis and Intracellular Transport of Nanoparticles: Present Knowledge and Need for Future Studies. Nano Today 2011, 6, 176-185.

(18) Jiang, W.; Kim, B. Y. S.; Rutka, J. T.; Chan, W. C. W. Nanoparticle-Mediated Cellular Response Is Size-Dependent. Nat. Nanotechnol. 2008, 3, 145-150.

(19) Alarcón-Correa, M.; Walker, D.; Qiu, T.; Fischer, P. Nanomotors. Eur. Phys. J.: Spec. Top. 2016, 225, 2241-2254.

(20) Maeda, H.; Nakamura, H.; Fang, J. The Epr Effect for Macromolecular Drug Delivery to Solid Tumors: Improvement of Tumor Uptake, Lowering of Systemic Toxicity, and Distinct Tumor Imaging in Vivo. Adv. Drug Delivery Rev. 2013, 65, 71-79.

(21) Dai, Y.; Xu, C.; Sun, X.; Chen, X. Nanoparticle Design Strategies for Enhanced Anticancer Therapy by Exploiting the Tumour Microenvironment. Chem. Soc. Rev. 2017, 46, 3830-3852.

(22) Torchilin, V. Tumor Delivery of Macromolecular Drugs Based on the Epr Effect. Adv. Drug Delivery Rev. 2011, 63, 131-135.

(23) Esteban-Fernández de Ávila, B.; Angell, C.; Soto, F.; LopezRamirez, M. A.; Báez, D. F.; Xie, S.; Wang, J.; Chen, Y. Acoustically 
Propelled Nanomotors for Intracellular Sirna Delivery. ACS Nano 2016, 10, 4997-5005.

(24) Xuan, M.; Shao, J.; Lin, X.; Dai, L.; He, Q. Self-Propelled Janus Mesoporous Silica Nanomotors with sub-100 nm Diameters for Drug Encapsulation and Delivery. ChemPhysChem 2014, 15, 2255-2260.

(25) Dobrovolskaia, M. A.; McNeil, S. E. Immunological Properties of Engineered Nanomaterials. Nat. Nanotechnol. 2007, 2, 469.

(26) Wilson, D. A.; Nolte, R. J. M.; van Hest, J. C. M. Autonomous Movement of Platinum-Loaded Stomatocytes. Nat. Chem. 2012, 4, 268.

(27) Tu, Y.; Peng, F.; André, A. A.; Men, Y.; Srinivas, M.; Wilson, D. A. Biodegradable Hybrid Stomatocyte Nanomotors for Drug Delivery. ACS Nano 2017, 11, 1957-1963.

(28) Tu, Y.; Peng, F.; Adawy, A.; Men, Y.; Abdelmohsen, L. K.; Wilson, D. A. Mimicking the Cell: Bio-Inspired Functions of Supramolecular Assemblies. Chem. Rev. 2016, 116, 2023-2078.

(29) Abdelmohsen, L. K.; Nijemeisland, M.; Pawar, G. M.; Janssen, G. J.; Nolte, R. J.; van Hest, J. C.; Wilson, D. A. Dynamic Loading and Unloading of Proteins in Polymeric Stomatocytes: Formation of an Enzyme-Loaded Supramolecular Nanomotor. ACS Nano 2016, 10, $2652-2660$.

(30) Kim, K. T.; Zhu, J.; Meeuwissen, S. A.; Cornelissen, J. J. L. M.; Pochan, D. J.; Nolte, R. J. M.; van Hest, J. C. M. Polymersome Stomatocytes: Controlled Shape Transformation in Polymer Vesicles. J. Am. Chem. Soc. 2010, 132, 12522-12524.

(31) Wilson, D. A.; Nolte, R. J.; Van Hest, J. C. Autonomous Movement of Platinum-Loaded Stomatocytes. Nat. Chem. 2012, 4, 268.

(32) Kim, K. T.; Zhu, J.; Meeuwissen, S. A.; Cornelissen, J. J.; Pochan, D. J.; Nolte, R. J.; van Hest, J. C. Polymersome Stomatocytes: Controlled Shape Transformation in Polymer Vesicles. J. Am. Chem. Soc. 2010, 132, 12522-12524.

(33) Abdelmohsen, L. K.; Williams, D. S.; Pille, J.; Ozel, S. G.; Rikken, R. S.; Wilson, D. A.; van Hest, J. C. Formation of WellDefined, Functional Nanotubes Via Osmotically Induced Shape Transformation of Biodegradable Polymersomes. J. Am. Chem. Soc. 2016, 138, 9353-9356.

(34) Rikken, R. S.; Engelkamp, H.; Nolte, R.; Maan, J.; Van Hest, J.; Wilson, D.; Christianen, P. Shaping Polymersomes into Predictable Morphologies Via out-of-Equilibrium Self-Assembly. Nat. Rev. Cancer 2016, 7, 12606.

(35) Tu, Y. F.; Peng, F.; Sui, X. F.; Men, Y. J.; White, P. B.; van Hest, J. C. M.; Wilson, D. A. Self-Propelled Supramolecular Nanomotors with Temperature-Responsive Speed Regulation. Nat. Chem. 2017, 9, 480-486.

(36) Men, Y.; Li, W.; Janssen, G.-J.; Rikken, R. S. M.; Wilson, D. A. Stomatocyte in Stomatocyte: A New Shape of Polymersome Induced Via Chemical-Addition Methodology. Nano Lett. 2018, 18, 20812085.

(37) Seifert, U. Configurations of Fluid Membranes and Vesicles. Adv. Phys. 1997, 46, 13-137.

(38) Meeuwissen, S. A.; Kim, K. T.; Chen, Y.; Pochan, D. J.; van Hest, J. C. Controlled Shape Transformation of Polymersome Stomatocytes. Angew. Chem. 2011, 123, 7208-7211.

(39) Yanagisawa, M.; Imai, M.; Taniguchi, T. Shape Deformation of Ternary Vesicles Coupled with Phase Separation. Phys. Rev. Lett. 2008, 100, 148102.

(40) Bermudez, H.; Brannan, A. K.; Hammer, D. A.; Bates, F. S.; Discher, D. E. Molecular Weight Dependence of Polymersome Membrane Structure, Elasticity, and Stability. Macromolecules 2002, $35,8203-8208$.

(41) Ma, X.; Hortelão, A. C.; Patino, T.; Sanchez, S. Enzyme Catalysis to Power Micro/Nanomachines. ACS Nano 2016, 10, 9111-9122.

(42) Dey, K. K.; Zhao, X.; Tansi, B. M.; Méndez-Ortiz, W. J.; Córdova-Figueroa, U. M.; Golestanian, R.; Sen, A. Micromotors Powered by Enzyme Catalysis. Nano Lett. 2015, 15, 8311-8315.

(43) Patiño, T.; Feiner-Gracia, N.; Arqué, X.; Miguel-López, A.; Jannasch, A.; Stumpp, T.; Schäffer, E.; Albertazzi, L.; Sánchez, S.
Influence of Enzyme Quantity and Distribution on the SelfPropulsion of Non-Janus Urease-Powered Micromotors. J. Am. Chem. Soc. 2018, 140, 7896-7903.

(44) Wang, Y.; Caruso, F. Enzyme Encapsulation in Nanoporous Silica Spheres. Chem. Commun. 2004, 40, 1528-1529.

(45) Iyer, P. V.; Ananthanarayan, L. Enzyme Stability and Stabilization-Aqueous and Non-Aqueous Environment. Process Biochem. 2008, 43, 1019-1032.

(46) Combes, D.; Yoovidhya, T.; Girbal, E.; Willemot, R. M.; Monsan, P. Mechanism of Enzyme Stabilization. Ann. N. Y. Acad. Sci. 1987, 501, 59-62.

(47) Frokjaer, S.; Otzen, D. E. Protein Drug Stability: A Formulation Challenge. Nat. Rev. Drug Discovery 2005, 4, 298.

(48) Chirino, A. J.; Ary, M. L.; Marshall, S. A. Minimizing the Immunogenicity of Protein Therapeutics. Drug Discovery Today 2004, 9, 82-90.

(49) Howse, J. R.; Jones, R. A. L.; Ryan, A. J.; Gough, T.; Vafabakhsh, R.; Golestanian, R. Self-Motile Colloidal Particles: From Directed Propulsion to Random Walk. Phys. Rev. Lett. 2007, 99, 048102 .

(50) Peng, F.; Men, Y. J.; Tu, Y. F.; Chen, Y. M.; Wilson, D. A. Nanomotor-Based Strategy for Enhanced Penetration across Vasculature Model. Adv. Funct. Mater. 2018, 28, 1706117.

(51) Gao, W.; Sattayasamitsathit, S.; Uygun, A.; Pei, A.; Ponedal, A.; Wang, J. Polymer-Based Tubular Microbots: Role of Composition and Preparation. Nanoscale 2012, 4, 2447-2453.

(52) Ma, X.; Sánchez, S. Bio-Catalytic Mesoporous Janus NanoMotors Powered by Catalase Enzyme. Tetrahedron 2017, 73, 48834886.

(53) Ortiz-Rivera, I.; Mathesh, M.; Wilson, D. A. A Supramolecular Approach to Nanoscale Motion: Polymersome-Based Self-Propelled Nanomotors. Acc. Chem. Res. 2018, 51, 1891-1900.

(54) Li, J.; Huang, G.; Ye, M.; Li, M.; Liu, R.; Mei, Y. Dynamics of Catalytic Tubular Microjet Engines: Dependence on Geometry and Chemical Environment. Nanoscale 2011, 3, 5083-5089.

(55) Solovev, A. A.; Mei, Y.; Bermúdez Ureña, E.; Huang, G.; Schmidt, O. G. Catalytic Microtubular Jet Engines Self-Propelled by Accumulated Gas Bubbles. Small 2009, 5, 1688-1692.

(56) Huang, G.; Wang, J.; Mei, Y. Material Considerations and Locomotive Capability in Catalytic Tubular Microengines. J. Mater. Chem. 2012, 22, 6519-6525.

(57) Ellis, L. M.; Hicklin, D. J. Vegf-Targeted Therapy: Mechanisms of Anti-Tumour Activity. Nat. Rev. Cancer 2008, 8, 579.

(58) Yancopoulos, G. D.; Davis, S.; Gale, N. W.; Rudge, J. S.; Wiegand, S. J.; Holash, J. Vascular-Specific Growth Factors and Blood Vessel Formation. Nature 2000, 407, 242.

(59) Bae, Y. H.; Park, K. Targeted Drug Delivery to Tumors: Myths, Reality and Possibility. J. Controlled Release 2011, 153, 198.

(60) Barua, S.; Mitragotri, S. Challenges Associated with Penetration of Nanoparticles across Cell and Tissue Barriers: A Review of Current Status and Future Prospects. Nano Today 2014, 9, 223-243.

(61) Hobbs, S. K.; Monsky, W. L.; Yuan, F.; Roberts, W. G.; Griffith, L.; Torchilin, V. P.; Jain, R. K. Regulation of Transport Pathways in Tumor Vessels: Role of Tumor Type and Microenvironment. Proc. Natl. Acad. Sci. U. S. A. 1998, 95, 4607.

(62) Gradishar, W. J.; Tjulandin, S.; Davidson, N.; Shaw, H.; Desai, N.; Bhar, P.; Hawkins, M.; O'Shaughnessy, J. Phase III Trial of Nanoparticle Albumin-Bound Paclitaxel Compared with Polyethylated Castor Oil-Based Paclitaxel in Women with Breast Cancer. J. Clin. Oncol. 2005, 23, 7794-7803.

(63) Chono, S.; Tanino, T.; Seki, T.; Morimoto, K. Uptake Characteristics of Liposomes by Rat Alveolar Macrophages: Influence of Particle Size and Surface Mannose Modification. J. Pharm. Pharmacol. 2007, 59, 75-80.

(64) Jiang, W.; Kim, B. Y.; Rutka, J. T.; Chan, W. C. NanoparticleMediated Cellular Response Is Size-Dependent. Nat. Nanotechnol. 2008, 3, 145 . 
(65) Lu, F.; Wu, S.-H.; Hung, Y.; Mou, C.-Y. Size Effect on Cell Uptake in Well-Suspended, Uniform Mesoporous Silica Nanoparticles. Small 2009, 5, 1408-1413.

(66) Lam, F.; Cladière, D.; Guillaume, C.; Wassmann, K.; Bolte, S. Super-Resolution for Everybody: An Image Processing Workflow to Obtain High-Resolution Images with a Standard Confocal Microscope. Methods 2017, 115, 17-27.

(67) Martinez-Outschoorn, U. E.; Lin, Z.; Trimmer, C.; Flomenberg, N.; Wang, C.; Pavlides, S.; Pestell, R. G.; Howell, A.; Sotgia, F.; Lisanti, M. P. Cancer Cells Metabolically "Fertilize" the Tumor Microenvironment with Hydrogen Peroxide, Driving the Warburg Effect. Cell Cycle 2011, 10, 2504-2520. 